\title{
Noncoding Subgenomic Flavivirus RNA: Multiple Functions in West Nile Virus Pathogenesis and Modulation of Host Responses
}

\author{
Justin A. Roby ${ }^{1, \dagger}$, Gorben P. Pijlman ${ }^{2, \dagger}$, Jeffrey Wilusz ${ }^{3}$ and Alexander A. Khromykh ${ }^{1, *}$ \\ 1 Australian Infectious Disease Research Centre, School of Chemistry and Molecular Biosciences, \\ The University of Queensland, St. Lucia, Brisbane 4072, Australia; E-Mail: j.roby@uq.edu.au \\ 2 Laboratory of Virology, Wageningen University, Wageningen, 6708NW, The Netherlands; \\ E-Mail: gorben.pijlman@wur.nl \\ 3 Department of Microbiology, Immunology \& Pathology, Colorado State University; Fort Collins, \\ CO 80523, USA; E-Mail: jeffrey.wilusz@colostate.edu
}

$\uparrow$ These authors contributed equally to this work.

* Author to whom correspondence should be addressed; E-Mail: alexander.khromykh@uq.edu.au; Tel.: +61-7-334-67219.

Received: 19 September 2013; in revised form: 13 January 2014 / Accepted: 15 January 2014 / Published: 27 January 2014

\begin{abstract}
Flaviviruses are a large group of positive strand RNA viruses transmitted by arthropods that include many human pathogens such as West Nile virus (WNV), Japanese encephalitis virus (JEV), yellow fever virus, dengue virus, and tick-borne encephalitis virus. All members in this genus tested so far are shown to produce a unique subgenomic flavivirus RNA (sfRNA) derived from the $3^{\prime}$ untranslated region (UTR). sfRNA is a product of incomplete degradation of genomic RNA by the cell 5'-3' exoribonuclease XRN1 which stalls at highly ordered secondary RNA structures at the beginning of the 3'UTR. Generation of sfRNA results in inhibition of XRN1 activity leading to an increase in stability of many cellular mRNAs. Mutant WNV deficient in sfRNA generation was highly attenuated displaying a marked decrease in cytopathicity in cells and pathogenicity in mice. sfRNA has also been shown to inhibit the antiviral activity of IFN- $\alpha / \beta$ by yet unknown mechanism and of the RNAi pathway by likely serving as a decoy substrate for Dicer. Thus, sfRNA is involved in modulating multiple cellular pathways to facilitate viral pathogenicity; however the overlying mechanism linking all these multiple functions of sfRNA remains to be elucidated.
\end{abstract}


Keywords: flavivirus; sfRNA; replication; pathogenicity; interferon; RNAi; XRN1

\section{Conservation of sfRNA in the Genus Flavivirus}

\subsection{Genus Flavivirus}

West Nile virus (WNV) is an emerging pathogen of humans that has the capacity to induce fatal encephalitis in the infected host [1-3]. WNV is a member of the genus Flavivirus within the family Flaviviridae which comprises small, enveloped viruses with non-segmented genomes consisting of single-stranded positive sense RNA. The Flavivirus genus can be divided into four main groups: mosquito-borne, tick-borne, no known vector (NKV), and the highly divergent insect-specific (ISF) groups (Figure 1A,B) [4-8]. Viruses belonging to the mosquito- and tick-borne groups are maintained in a natural transmission cycle between amplifying vertebrate hosts and particular haematophagous arthropods. NKV viruses are restricted to mammalian bat or rodent hosts, without a known arthropod vector for transmission. ISFs are the most divergent group, infecting and transmitted by predominantly Aedes and Culex genus mosquitos, and are unable to infect vertebrate hosts. Tamana bat virus (TABV) is the most divergent species apparently replicating exclusively in mammalian cells [9], requiring placement in its own group removed from the rest of the genus [10,11].

\subsection{The 3'UTR of the Family Flaviviridae}

The family Flaviviridae is composed of viruses with monocistronic single-stranded positive-polarity RNA genomes that lack a polyA tail. Flanking the single ORF are highly structured 5' and 3' untranslated regions (UTRs) that act as important mediators of viral genome replication and translation. These UTRs contain conserved and highly elaborated secondary structures that are generally more pronounced at either end of the genome [12]. Viruses of the genera Hepacivirus, Pegivirus, and Pestivirus concentrate the highest degree of structural elaboration at their 5'UTRs which have evolved to function as internal ribosome entry sites (IRES) that drive cap-independent translation of the polyprotein. Members of the genus Flavivirus, to which WNV belongs, retain an $\mathrm{m}^{7} \mathrm{GpppAmpN} \mathrm{N}_{1}$ cap structure at the 5'-terminus and thus have shorter 5'UTRs $(\approx 100 \mathrm{nt})$ [13] consisting of a pair of conserved stem-loops (SL-A and SL-B) [14] and encoding regions important for genome cyclisation at the initial phase of replication (5'CS, 5'UAR, and 5'DAR; reviewed in [15]).

The 3'UTR of flaviviruses is considerably larger ( $\approx 380-600 \mathrm{nt}$ in length) and can - in the case of mosquito-borne flaviviruses - be divided into three domains: a highly variable proximal domain 1 that directly follows the stop codon, a second domain 2 with moderately conserved sequence and a number of stem-loop and dumbbell structures, and the highly conserved distal domain 3 which contains the complementary cyclisation elements and the stable, terminal stem-loop structure (Figure 2A) [15]. The 3'UTR of WNV consists of an AU-rich stem-loop SL-I structure followed by a highly-conserved, branched, pseudoknot (PK1)-forming SL-II immediately preceding a short conserved loop RCS3 (Figure 2A). This structural motif is then effectively repeated by SL-III, SL-IV (with PK2) and CS3 (Figure 2A). Downstream of this are two dumbbell structures DB1 and DB2, with DB1 also predicted 
to form pseudoknot PK3 (Figure 2A). This region is then followed by a predicted short stem-loop and the large terminal 3'SL (Figure2A) [15-18].

Figure 1. Phylogenetic relationships and sfRNA production within the genus Flavivirus and the family Flaviviridae. (A) Phylogenetic tree demonstrating the evolutionary relationships between flaviviruses characterised for sfRNA production and basal Tamana bat virus (TABV) which remains uncharacterised (underlined). The tree was generated using the amino acid sequence of the entire genomic polyproteins. Vector classes for virus groups are indicated by black bars to the right of the tree. Primary vertebrate hosts are indicated in italics (other than for ISFs). Bar $=0.2$ substitutions per site. ISF $=$ insect-specific flavivirus; $\mathrm{NKV}=$ no known vector. Modified from Cook et al., 2012 and Kitchen et al., 2011 [5,6]; (B) sfRNA does not appear to be produced by other genera (Hepacivirus, Pestivirus) within the family Flaviviridae. The proposed genus Pegivirus remains uncharacterised for sfRNA production. Phylogenetic tree generated using the amino acid sequences of viral RNA-dependant RNA polymerases. Bar $=0.2$ substitutions per site. Modified from Stapleton et al., 2011 [11].

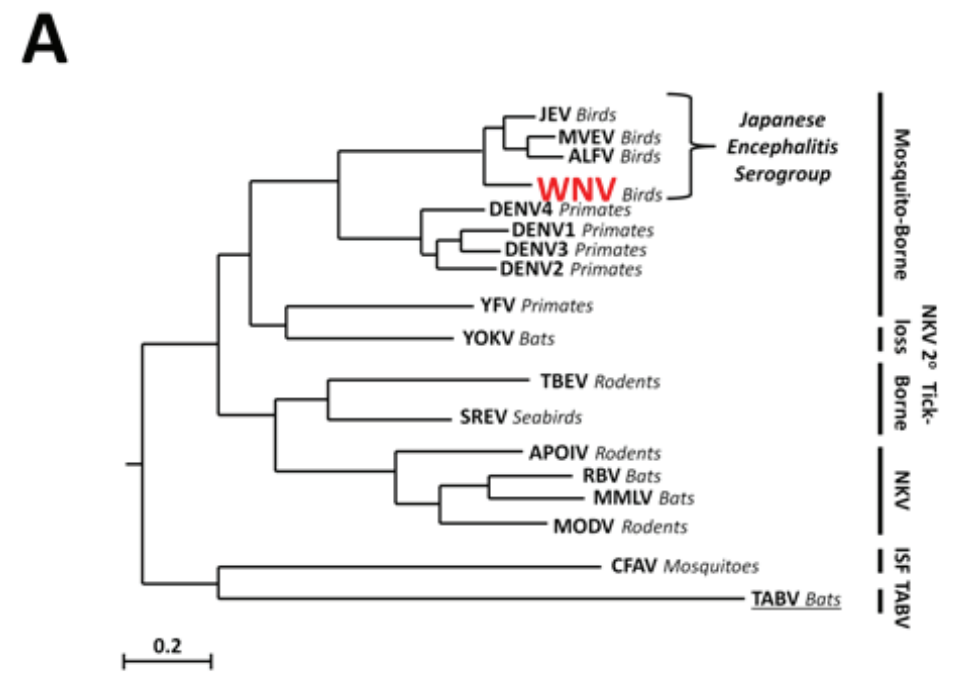

B

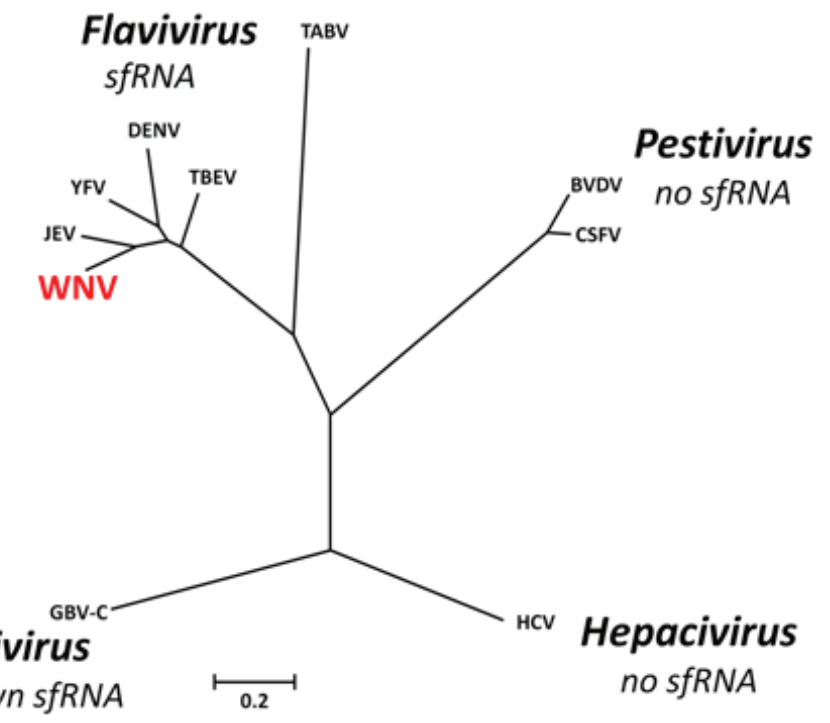


Figure 2. Structural elements within the 3'UTR of WNV and conservation of SL-II within the genus Flavivirus. (A) Schematic representation of the 3'UTR of WNV demonstrating the arrangement of stem-loops (SL) and pseudoknots (PK) and the sfRNA 5'-terminus. Modified from Pijlman et al., 2008 [18] and Funk et al., 2010 [19]; (B) Schematic representation demonstrating the predicted conservation of the SL-II/PK1-like RNA structure within the 3'UTR of divergent members of the genus Flavivirus.

A

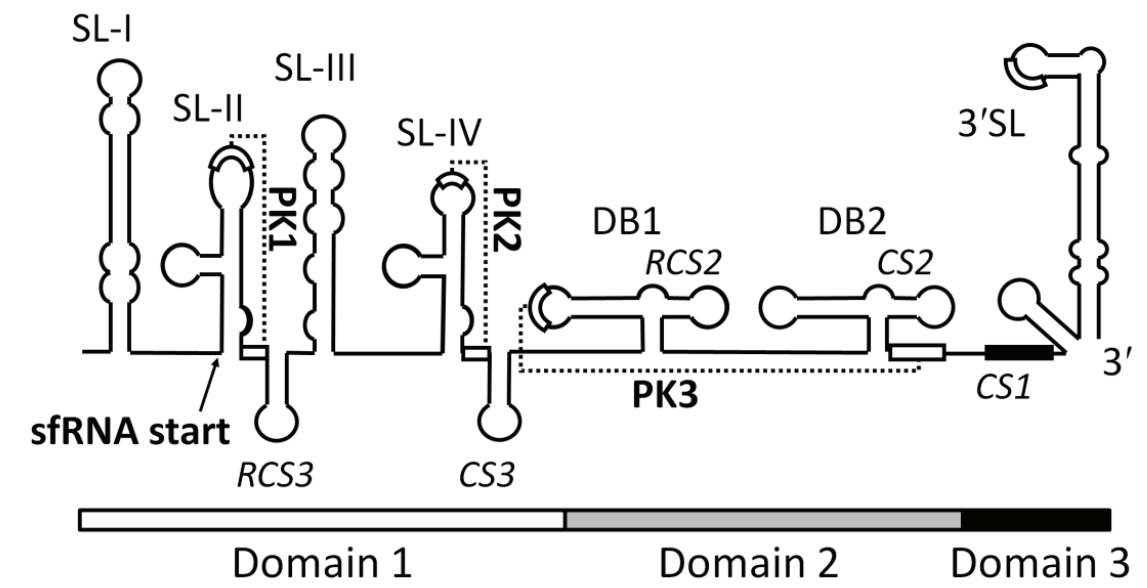

B

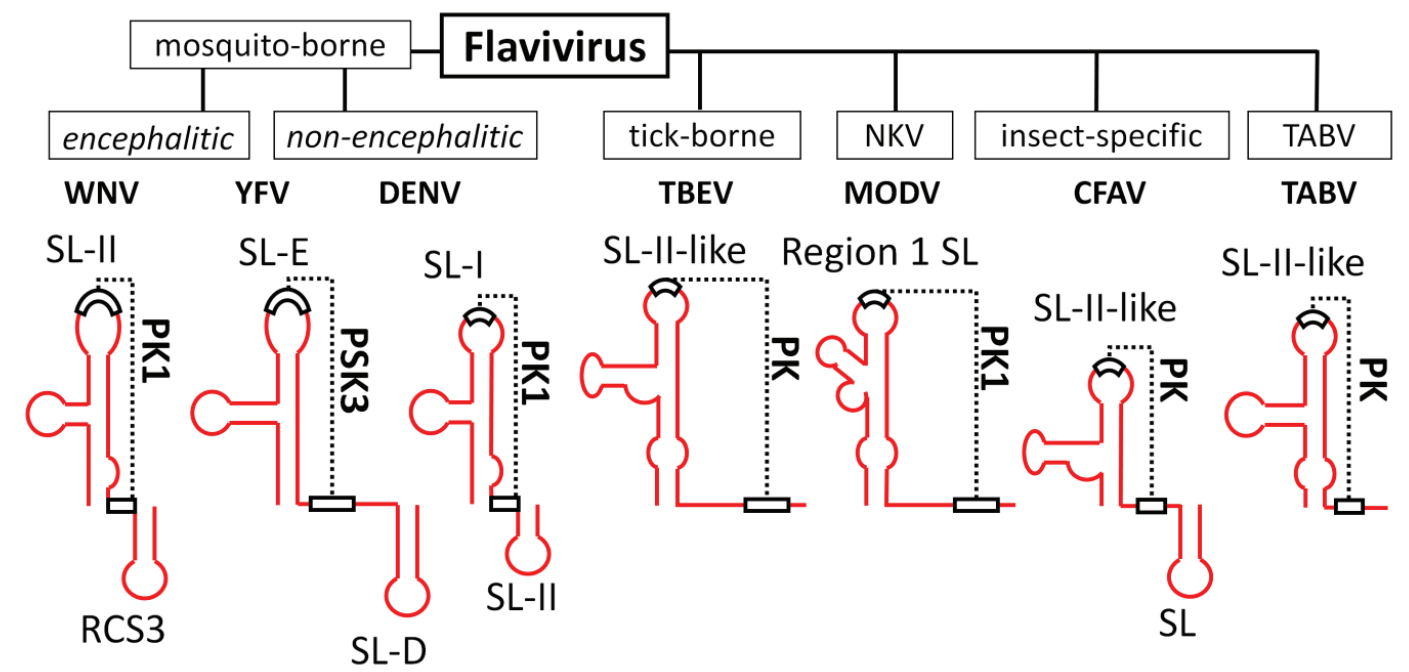

The 3'UTR of flaviviruses has been demonstrated to bind to several host proteins (see the last section) as well as proteins of the viral replication complex (RC). Such interactions promote genomic RNA cyclisation which is important for two distinct functions: (i) SL-A in the 5'UTR acts as a promoter element [20-22] that stimulates the viral RNA-dependent RNA polymerase NS5 to begin negative strand synthesis at the 3 'UTR, initiating the replication cycle [15], and (ii) the 3'UTR binds to host proteins that normally associate with mRNA polyA tails such as phosphorylated translation elongation factor $1 \alpha(\mathrm{EF}-1 \alpha)$ and poly(A)-binding protein (PABP), thus bringing the 5' and 3'UTRs into close association, which allows cap-dependent translation of the viral polyprotein to proceed [15,23-25]. 


\subsection{Discovery of sfRNA}

The accumulation of a small viral RNA species now known as sfRNA was first observed for the mosquito-borne encephalitic flaviviruses Murray Valley encephalitis virus (MVEV) [26], Japanese encephalitis virus (JEV) [27], and WNV [28]. These early observations identified the presence of the sfRNA species in vitro and in vivo without ascribing a biological role to the moiety, although in the JEV paper [27] the authors mapped sfRNA to the 3'UTR and determined its 5' end. Although attempts were undertaken to explain the nature and generation mechanism of the molecule, this remained elusive until 2008 when we first reported the mechanism of sfRNA generation and the important role of sfRNA in pathogenicity of WNV [18].

In this 2008 manuscript we utilized an extensive array of recombinant constructs expressing truncated lengths of the WNV genome to demonstrate that sfRNA is a product of the 3'UTR, not requiring the presence of other gRNA sequences, viral proteins or active viral replication. We identified cellular 5'-3' exoribonuclease XRN1 as the enzyme responsible for the generation of sfRNA via stalling at stem-loop (SL) structures while degrading viral gRNA. Similar to the JEV study [27], we used primer extension to map the 5'-terminus of WNV sfRNA to the beginning of SL-II structure.

A series of deletion mutants demonstrated the importance of secondary structures within the 3'UTR in sfRNA generation with mutant viruses incapable of producing full-length sfRNA species, sfRNA1, exhibiting decreased cytopathicity in cells and attenuation of virulence in a murine model of infection.

\subsection{Conservation of sfRNA between Different Groups within the Genus Flavivirus}

The outcomes of the 2008 WNV sfRNA paper and several studies since then [18,29-31] were that sfRNA was demonstrated to be accumulating concurrently with genomic RNA in both vertebrate and invertebrate cells (Figure 3A) infected with members of the genus Flavivirus from each of the mosquito-borne (encephalitic: WNV, MVEV, Alfuy virus; non-encephalitic: Dengue virus (DENV) and Yellow fever virus (YFV)) and tick-borne (Samaurez Reef virus) groups, and absent from the genera Hepacivirus (HCV replicon) and Pestivirus (Bovine viral diarrhoea virus). Indeed, sfRNA has been demonstrated to be produced in infections with every member of the genus Flavivirus that has subsequently been assessed, expanding the cohort of sfRNA producers to include NKV viruses (Modoc virus (MODV), Apoi virus, Rio Bravo virus, Montana myotis leukoencephalitis virus, and Yokose virus) [32] and ISFs (cell-fusing agent virus (CFAV), Culex flavivirus (CxFV)) [33], as well as tick-borne encephalitis virus (TBEV) [34].

Importantly there remain at least two groups within the family Flaviviridae that have not been assessed for sfRNA production. As the most basal and distantly related member of the genus Flavivirus [10] it would be interesting to investigate sfRNA formation by TABV. Indeed, RNA modeling of a 241-nt sequence downstream of the predicted TABV NS5 coding sequence (partial 3'UTR; Genbank accession AF346759.1) did show the presence of a SL-II-like structure with a 4 bp-pseudoknot interaction but without downstream stemloop (Figure 2B), yet the predicted length of the resulting putative sfRNA molecule is not known until the complete 3'UTR has been sequenced. If sfRNA is experimentally demonstrated to be produced by TABV it would confirm that this moiety is characteristic of the genus, however if it is found to not be produced by this virus, this may indicate that sfRNA 
solely emerged as a means to facilitate infection of invertebrate hosts. This last possibility is intriguing given that sfRNA has been demonstrated to inhibit the predominant insect innate immune pathway, RNA interference (RNAi; see below) [35], and that members of the genus Flavivirus are the only viruses in the entire family Flaviviridae to productively infect invertebrates. The other group of viruses yet to be investigated are the members of the genus Pegivirus (e.g., GB virus C). Several conserved RNA secondary structures have been predicted within the 3'-terminus of pegivirus genomes, more so than that observed in hepaciviruses and pestiviruses [12]. Thus the possibility of sfRNA-like production in this genus may shed light on the evolutionary relationships of viruses in this family.

\section{Mechanism of sfRNA Generation}

\subsection{Exoribonuclease Stalling}

The unique sfRNA generation mechanism involves the efficient stalling of 5'-3' exoribonuclease XRN1 during degradation of viral genomic RNA [18,19,30,35]. XRN1 is a key enzyme in host and viral mRNA turnover [36,37] occurring in cytoplasmic processing bodies (PBs) [36] and is a classical PB marker [38]. The stalling of XRN1 in flavivirus RNA is caused by a short RNA structure of $\sim 80 \mathrm{nt}$ at the $5^{\prime}$ end of sfRNA (called SL-II in WNV or SL-E in YFV) with high stability involving a pseudoknot $(\mathrm{PK})$ base pairing interaction between the upper loop and a short sequence downstream of the SL structure (PK1, Figure 2A). Although the primary sequence is highly variable, this SL-II/PK1 structure has been predicted to be remarkably well conserved in all flavivirus strains demonstrated to produce sfRNA [17,18,31] (Figure 2B). The WNV SL-II/PK1 sequence has also been successfully used to stall XRN1-mediated degradation of herpes virus RNA [39]. The 3D structure of SL-II/PK1 remains unknown but is most likely key to fully understand its functional ability to stall and neutralize XRN1.

\subsection{RNA Structures Required for sfRNA Production}

Initial research efforts to study the 3'UTR were mostly focused on sequences at the very 3'-terminus of gRNA [13,40-42] while the 5'end of the 3'UTR was simply regarded as the variable domain. Yet, it is in this 5 'end where one of the essential features of the 3'UTR is conserved, namely the RNA structures responsible for stalling of XRN1 $[18,19,30]$.

The contribution of each of the SL and PK structures in facilitating XRN1 stalling has been investigated for WNV [18,19] and YFV [30]. Experiments involving disruption of these secondary and tertiary structures in the genomes of both viruses provide compelling evidence for the absolute requirement of a pseudoknot in sfRNA generation. Straightforward deletion of SL-II in WNV (FL $\triangle$ SLII), mutation of the stem-loop (FL-IRA), or disruption of PK1 (FL-PK1') led to production of a smaller sfRNA species of approximately $365 \mathrm{nt}$ (sfRNA2) predicted to form by XRN1 stalling at the SL-IV/PK2 (analogous in structure to the upstream SL-II/PK1) (Figure 3B-D) [18,19]. Deletion of SL-E in YFV (YFV- $\Delta$ SLE), however, led to complete abrogation of sfRNA production as determined via Northern blot using a probe complimentary to sequences in SL-B at the very centre of sfRNA [30]. An analogous phenotype was observed upon mutation of the stem-loop, or disruption of the component PK. Unlike WNV, YFV does not have a repeated SL-E structure to act as a secondary stalling point; hence the 5'-3' degradation mediated by XRN1 may no longer be prevented. 
Figure 3. sfRNA is generated by XRN1 stalling at conserved SL and PK structures. (A) Northern blot detection of WNV sfRNA accumulation over the course of infection in mammalian (Vero) and mosquito (C6/36) cell lines using oligonucleotide probes complementary to the 3'SL [19]; (B) Schematic representation of the mechanism of sfRNA generation. Following decapping or upstream cleavage within the flavivirus gRNA, host XRN1 degrades 5'-3' stalling at the SL-II/PK1 structure and thus forming sfRNA1 and becoming inactive by association with this moiety. Mutation to delete/disrupt SL (C) or PK (D) structures leads to downstream XRN1 stalling and accumulation of successively smaller sfRNA species. Modified from Pijlman et al., 2008 [18] and Funk et al., 2010 [19].

A

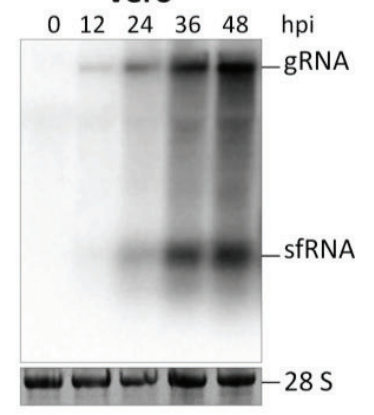

$\mathrm{C} 6 / 36$

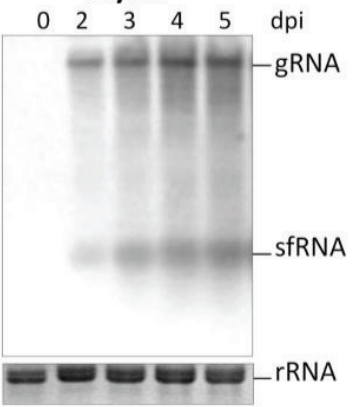

B

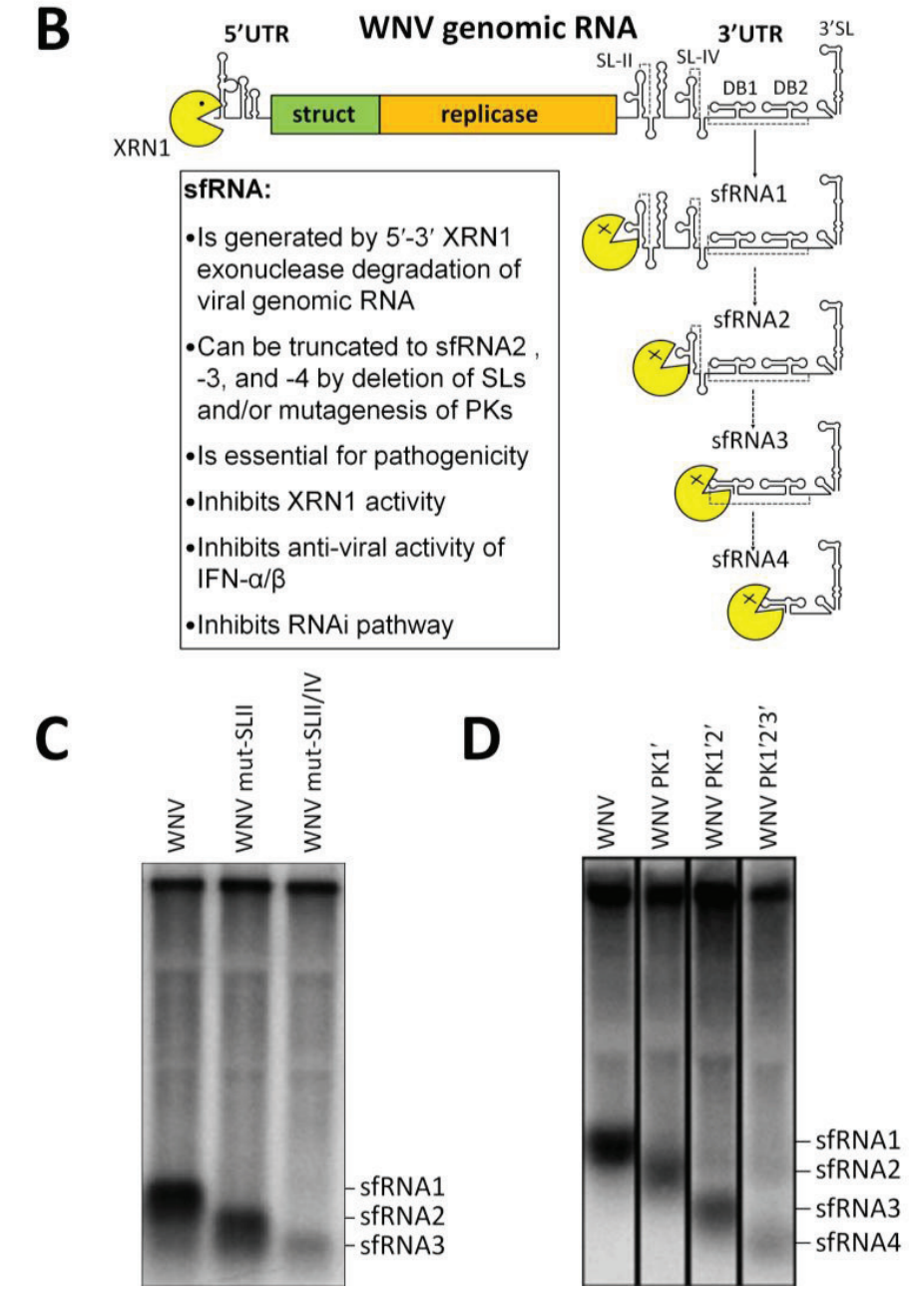

SL-II

3NTR $^{3}$ 3'SL $9\{:$ DB1 DB2 
The YFV 3'UTR does contain a further two predicted PKs, forming at downstream SLs, thus these too fail to stall XRN1. This contrasts with the utility of the PK interactions in the WNV 3'UTR. Further disruption of secondary/tertiary structure in WNV via deletion of SL-II to RCS3 and CS3 (FL $\triangle$ SLII-RCS3 $\triangle \mathrm{CS} 3$ ), disruption of SL-II and deletion of CS3 (FL-IRA $\Delta$ CS3), or mutation of PK1 and PK2 (FL-PK1'2') leads to production of a smaller sfRNA species of $\approx 270 \mathrm{nt}$ (sfRNA3) predicted to form by XRN1 stalling at DB1/PK3 (Figure 3B-D) [18,19]. An even smaller sfRNA4 produced presumably by stalling at DB2 was detected in mosquito cells infected with WNV containing combined mutations in upstream PKs 1, 2, and 3 (FL-PK1'2'3' in Figure 3B,D) [19].

In each example of deletions/mutations producing shorter sfRNA species the corresponding viruses were attenuated in regards to cytopathicity, and pathogenicity [18,19,30,43], demonstrating the importance of full-length sfRNA species, sfRNA1, to these processes. However several questions still remain to be answered in regards to the physical limitations of SL/PK interactions in XRN1 stalling. For instance, what is the minimal PK structure for stalling XRN1? What is the minimal SL-II structure? Can we learn from other viruses or different groups within the flaviviruses (e.g., ISFs)? Are these structures present in eukaryote genomes as well and, if so, do they facilitate a biological role?

\subsection{Endogenous Production of Truncated sfRNAs}

One notable aspect of sfRNA production that has received little attention is that infection with wild-type flavivirus isolates has been observed to lead to the natural production of truncated sfRNA species [19,29-31]. The presence of a smaller, less intense sfRNA band in a Northern blot is generally only observed occasionally for wild-type viruses and thus goes unmentioned in publications [19,29]. Such low intensity and infrequent bands likely represent occasional "slipping" of the XRN1 stalling process at SL-II. However, this occasional slipping does not appear to be the case with YFV and selected strains of DENV-2 [30,31].

Unlike the infrequent production/detection of smaller sfRNA species noted in other wild-type viruses, certain strains of DENV appear to readily produce these truncated species in infected cells [31]. This was particularly noted for the Chinese isolates of DENV-2 (strains DV2-FJ10 producing sfRNA1 and 2, and DV2-43 producing sfRNA1, 2, and 3) in infected BHK-21 cells. The three sfRNA species produced by DV2-43 were also readily observed in infected mouse brain and C6/36 mosquito cells. While the biological relevance of these truncations remains to be determined, it is important to note that DV2-43 is an isolate from an infected patient that displays no attenuated phenotypes in culture [44]. This observation contrasts with demonstrated attenuative effects of mutations in WNV leading to generation of shorter sfRNA species $[18,19,43]$ and may therefore indicate a differential requirement for full-length sfRNA in pathogenicity/virulence of different flaviviruses.

Through the use of ${ }^{32} \mathrm{P}$-labelled oligonucleotide probes complementary to sequences at the 5 '- and 3'-termini of sfRNA, a shorter YFV sfRNA2 was demonstrated to be a product of 3'-truncation in mammalian (but not insect C6/36) cell lines leading to deletion of the terminal 3'SL [30]. Notably this means of truncation appears unique to YFV as WNV does not produce this form of smaller sfRNA) [18] and the exact mechanism of this $3^{\prime}-5$ ' truncation is yet to be determined. 


\section{Cellular Localisation of sfRNA}

Accurate localisation of sfRNA in infected cells has been hampered by an inability to discriminate between the 3'UTR of gRNA and processed sfRNA in infected cells. Nevertheless, the use of fluorescence in-situ hybridisation (FISH) probes to specifically detect the WNV 3'UTR (sfRNA) and NS3 gene (gRNA) has demonstrated that sfRNA forms punctuate foci distinct from the perinuclear localisation of gRNA [45]. These foci co-localise variably with XRN1 (a marker of PBs) [18] and with the stress-granule (SG) marker protein eIF3y [45]. PBs are cytoplasmic aggregates that contain proteins involved in diverse posttranscriptional processes, such as mRNA degradation and RNA-mediated gene silencing [38]. The RNAi machinery is also concentrated in PBs [37]. PBs mediate the decapping and 5'-3' degradation of mRNA as part of the homeostatic turn-over of nucleic acids [36,37] and have been demonstrated to traffic some of their components to the sites of WNV replication [46]. Localisation of sfRNA to PBs is consistent with the generation of this RNA species [18], and our later studies determined that during its generation (presumably in PBs) sfRNA remains bound to and inactivates XRN1 which leads to increase in host mRNA half-lives [35] (see below). SGs accumulate during cellular response to various stress stimuli and consist of stalled translational pre-initiation complexes that act to temporarily suppress protein translation [37,47]. SGs can have diverse pro- and antiviral functions [47] and some viruses actively suppress bona fide SG assembly [48], however, the molecular interactions between viral products and SG components are only beginning to be elucidated. A biological role for sfRNA co-localisation with SGs has yet to be determined. As SGs act to sequester cellular RNA pools it is possible that sfRNA association is non-specific. It is worth noting however, that $\mathrm{WNV}$ has been demonstrated to actively delay the SG response by delaying early gRNA replication [49] and a functional role for sfRNA in this process cannot be discounted at this stage.

\section{Flavivirus Replication and sfRNA}

Decreased replication in mammalian and mosquito cell lines was observed for WNV and YFV mutants deficient in sfRNA $[18,19,30]$. A more recent study investigating the roles of sfRNA in JEV infection has revealed preliminary evidence that suggests sfRNA may play a role in viral RNA replication and/or translation [29]. Although the experimental methodology used in the JEV paper was at times highly artificial (sfRNA were transcribed in vitro from a T7 promoter thus are unlikely to possess an authentic 5'-monophosphate and so are unlikely to act genuinely in vivo), at least one key set of experiments lends credence to the authors' hypothesis. Transfection of JEV-infected BHK-21 cells with in vitro transcribed antisense sfRNA at $28 \mathrm{~h}$ post-infection (hpi) led to a notable increase in antisense gRNA at 38 and 48 hpi compared to cells either transfected with positive-sense sfRNA or mock-transfected [29]. While the biological activity described appears genuine, it is unclear exactly how this antisense sfRNA is promoting antigenome synthesis. It may base-pair with endogenous positive-sense sfRNA and so negate a possible role in antigenome suppression. Alternatively it may displace the 5'-end of the antigenome within the replicative form to allow NS5 access and new antigenome synthesis.

In addition, both positive-sense and antisense sfRNA were able to specifically inhibit translation of a JEV-based Renilla luciferase reporter minicon (Renilla luciferase gene incorporated in-frame after 
$\triangle$ capsid in a truncated JEV genome consisting of 5' and 3'UTRs, $\Delta$ capsid, $\Delta$ envelope, $\triangle \mathrm{NS} 1$, and $\triangle \mathrm{NS5}$ ) in vivo in transfected BHK-21 cells. Samples exposed to control RNA derived from JEV E-NS1 sequences did not demonstrate translation inhibition. This phenotype was also observed in vitro during incubation with rabbit reticulocyte lysate (RRL) [29]. Unfortunately, however, the results described will need to be supported by future experiments utilizing live viral infections as these minicon investigations do not simulate the context of viral gRNA expression with absolute authenticity. Without expression of the non-structural proteins and true genomic replication, the characteristic for flavivirus infection cellular membrane environment [50-52] is lacking which may have profound consequences for sfRNA and translation-factor access to gRNA.

\section{MicroRNA Production from sfRNA}

The predominant innate antiviral response in insects, RNA interference (RNAi), was first identified in plants and appears to be a conserved mechanism of innate immunity and epigenetic control in eukaryote organisms [53,54]. RNAi centres on the cleavage of dsRNA into 21-30 nt long ssRNA which is then loaded into RNA-induced silencing complexes (RISC) and utilised as a homing motif for base-pairing of target sequences which are subsequently cleaved, downregulated, or upregulated. RNAi is divided into three main branches: microRNA (miRNA) which is generally involved in epigenetic control of expression, small-interfering RNA (siRNA) involved in the antiviral response, and P-element-induced wimpy testes in Drosophila (PIWI)-associated interfering RNA (piRNA) which is utilised primarily for the control of mobile genetic elements [53,55]. RNAi has been demonstrated to be active in the insect immune response to WNV, contributing to the evolution of viral RNA diversity [56].

Canonical biogenesis of miRNA requires a nuclear step where drosha cleaves miRNA transcript into pri-miRNA [57,58], however, mounting evidence suggests the existence of a non-canonical cytoplasmic pathway of miRNA generation $[59,60]$. There have been only few reports of miRNA production by cytoplasmic RNA viruses, and most of these are engineered cellular miRNA precursors inserted in the viral genome of, e.g., TBEV [59] and SINV [61].

Recently we identified the first flavivirus-derived miRNA, KUN-miR-1, in WNV-infected mosquito cells [62]. WNV sfRNA was deemed the likely source of KUN-miR-1 as expression of this RNA species from a heterologous Semliki Forest virus (SFV, an unrelated alphavirus) replicon was sufficient to lead to production of the functional KUN-miR-1 miRNA. In addition, infection with FL-IRAdCS3 mutant WNV resulted in detection of diminished amounts of KUN-miR-1, although it also coincided with diminished viral RNA replication. KUN-miR-1 was demonstrated as important for the WNV lifecycle in mosquitoes, as a specific inhibitor of this miRNA greatly reduced virus replication in mosquito cells. In addition, a host mRNA target for KUN-miR-1 in mosquito cells was determined to be zinc-finger transcription factor GATA4. WNV infection of or SFV-sfRNA replication in mosquito cells upregulated the levels of GATA4 mRNA so did the ectopic expression of pre-KUN-miR-1 alone from a plasmid DNA or from SFV replicon. Importantly, knock-down of GATA4 led to a reduction in WNV replication analogous to that observed with a KUN-miR-1 inhibitor. While the exact nature of the WNV interaction with GATA4 remains unknown, this host protein has been linked in mosquitoes to the trafficking of lipids [62]. Thus it is possible that KUN-mirR-1-induced up-regulation of GATA4 
plays a pivotal role in facilitating proliferation of a lipid-rich virus-induced membrane environment known to be crucial for flavivirus RNA replication [50-52].

\section{Host Response and sfRNA}

\subsection{Cytopathicity in Cells and Pathogenicity in Mice are Dependent on sfRNA}

Early experiments to characterise the activities of sfRNA demonstrated that the cytopathic effect (CPE) generated by WNV mutants that produced less abundant, truncated sfRNAs were significantly less pronounced in Vero cells, leading to marked reduction in size or complete absence of viral plaques [18]. This loss of CPE was observed for mutants containing either deletions in SL structures [18] or disruption of PK interactions [19]. Importantly, transfection of Vero cells with a plasmid designed to produce authentic sfRNA upon XRN1-mediated degradation (pCMVßgal3') partially rescued CPE of the sfRNA1-deficient FL-IRA $\triangle \mathrm{CS} 3$ virus [18]. This phenotype can be specifically attributed to sfRNA as transfection of the control plasmid pCMVßgal did not demonstrate recovery of CPE during FL-IRA $\triangle \mathrm{CS} 3$ infection.

This reduction in CPE appears to directly correlate with attenuation of WNV virulence in a murine model of infection. Three week old mice injected intraperitoneally (i.p.) with 10,000 plaque-forming units (PFU) of both FL-IRA (sfRNA2) and FL-IRA $\triangle \mathrm{CS} 3$ (sfRNA3) failed to demonstrate symptomatic infection and remained alive by 14 days post-infection (dpi) [18]. This is in contrast to mice that received the same dose of wild-type Kunjin strain of WNV (FLSDX) which all succumbed to infection by 9 dpi. These results have been recapitulated with PK mutants: i.p. injection of three week old mice with 10,000 PFU of mutant viruses FL-PK1', FL-PK1'2', and FL-PK1'2'3' failed to induce mortality greater than $20 \%$ by 14 dpi [19] while infection of mice with wild type FLSDX virus led to complete mortality by 8 dpi in this experiment. Interestingly, all mutant viruses despite demonstrating high degree of attenuation of virulence were effective in eliciting an immune response that provided complete protection against lethal challenge with highly pathogenic New York 99 strain of WNV. Thus, mutations leading to deficiency in generation of sfRNA1 can be employed in developing effective live attenuated flavivirus vaccines.

\subsection{The Interferon Response and $s f R N A$}

In order to characterise the parameters leading to the reduced pathogenicity in vivo observed for sfRNA-deficient WNV mutants $[18,19]$, the potential role of the host type-I interferon (IFN- $\alpha / \beta)$ response was investigated. The IFN- $\alpha / \beta$ response pathway has been demonstrated as the most important mediator of host resistance to flavivirus infection $[63,64]$. Thus mutations that lead to virus attenuation are likely to affect the viral countermeasures to IFN- $\alpha / \beta$.

Infection of wild-type (IFN-competent) mouse embryonic fibroblasts (MEFs) at a multiplicity of infection (MOI) of 1 with sfRNA-deficient FL-IRA $\triangle \mathrm{CS} 3$ led to decrease in gRNA replication and virion formation compared to FLSDX infection as measured by Northern blot and plaque assay, respectively [43]. In contrast, infection of MEFs with knock-out of the IFN regulatory factor (IRF)-3 and -7 genes (IRF-3/ $/ 7^{--}$; cannot effectively produce IFN- $\alpha / \beta$ but can respond to exogenous IFN) demonstrated no appreciable difference in replication efficiency between FL-IRA $\triangle \mathrm{CS} 3$ and FLSDX viruses. 
The relationship between full-length sfRNA production and viral subversion of the host IFN- $\alpha / \beta$ response was further confirmed by complementary experiments assessing the effects of addition or neutralisation of IFN upon WNV replication. Pre-incubation of IRF-3/7 $7^{-/}$MEFs with increasing concentrations of exogenous IFN- $\alpha$ followed by infection with FL-IRA $\Delta$ CS3 and FLSDX viruses demonstrated a significantly higher sensitivity of the sfRNA-deficient mutant to the anti-viral activity of IFN- $\alpha$. Conversely, neutralisation of the IFN- $\alpha / \beta$ receptor IFNAR 1 by monoclonal antibodies during infection was able to rescue replication of FL-IRA $\triangle \mathrm{CS} 3$ mutant virus in wild-type MEFs [43].

In vivo experiments confirmed these results by comparing infection of wild-type C57BL/6 mice with IRF-3/ $7^{-/-}$mice. The results demonstrated increased virulence of FL-IRA $\Delta$ CS3 in knock-out mice with $80 \%$ mortality by 9 dpi with $10^{3}$ PFU of virus (a dose that was only able to kill $50 \%$ of wild-type mice). Additional infections of IFNAR $^{-/}$mice demonstrated that FL-IRA $\triangle$ CS3 was universally lethal by 8 dpi when injected with $10^{3}$ PFU virus (a delay of only 2-3 days compared to FLSDX). Assessment of viraemia by qRT-PCR however, demonstrated that FL-IRA $\triangle \mathrm{CS} 3$ replication, while increased in $\mathrm{IFNAR}^{-/-}$mice, was still significantly reduced compared to FLSDX [43] indicating a potential partial contribution of IFN- $\alpha / \beta$-independent host responses in controlling mutant virus replication in the mouse model of WNV infection.

Although sfRNA had convincingly been demonstrated to subvert host IFN- $\alpha / \beta$ signalling, the exact mechanism involved in this process had yet to be elucidated. IFN- $\alpha / \beta$ signalling in mammalian cells induces the nuclear translocation of phosphorylated signalling transducer and activator of transcription (STAT)-1 and -2 proteins and the upregulated expression of hundreds of antiviral IFN-stimulated genes (ISGs) $[63,64]$. In order to gauge the influence of sfRNA production on ISG mode of action, two well characterised ISGs known to exhibit activity during WNV replication-protein kinase R (PKR) [65-68] and RNase L [28,66,69] — were investigated for their ability to be modulated by sfRNA.

PKR has many potential roles in eukaryote cells including anti-proliferative, cell death, inflammatory, and innate immune activities. PKR recognises dsRNA of at least $30 \mathrm{nt}$ in length, but optimally 70-80 nt [70]. The antiviral activity of PKR is predominantly mediated via phosphorylation of the $\alpha$-subunit of eukaryote initiation factor 2 (eIF2 $\alpha$ ) at serine 51 which ultimately inhibits mRNA translation [70]. $\mathrm{PKR}^{-/}$MEFs demonstrated no observable rescue of FL-IRA $\triangle \mathrm{CS} 3$ replication compared to that observed via infection in wild-type MEFs [43], thus PKR is unlikely to be inhibited by sfRNA.

RNase $\mathrm{L}$ is a ssRNA-specific endonuclease activated by the binding of 2'-5'-linked oligoadenylates $\left(2^{\prime}-5 A_{n}\right)$; a unique molecule produced by the 2'-5'-oligoadenylate synthetase (OAS) family of proteins upon the binding of dsRNA and stem-loops $>15 \mathrm{nt}$ in length [70]. RNase L is thought to exert antiviral activity via the direct degradation of gRNA as well as by cleaving host mRNAs to generate novel IFN-stimulating moieties [70]. In contrast to results obtained in $\mathrm{PKR}^{-/}$MEFs FL-IRA $\triangle \mathrm{CS} 3$ replication was partially rescued in RNase $\mathrm{L}^{-/}$MEFs, indicating a potential interaction of sfRNA with the 2'-5'-oligoadenylate synthetase (OAS)/RNase L pathway [43]. However an in vitro assay demonstrated that sfRNA does not associate directly with RNase L as it was unable to prevent this endonuclease from degrading WNV gRNA or other RNase L-sensitive viral RNAs. Virulence of FL-IRA $\Delta$ CS3 mutant was also not rescued in RNase $\mathrm{L}^{-/-}$mice further indicating that RNase $\mathrm{L}$ is unlikely to be a direct sfRNA target.

Preliminary evidence has also demonstrated that transfection of in vitro transcribed sfRNA may inhibit IRF-3 phosphorylation in JEV-infected cells [71]. The authors propose that this inhibition of 
IRF-3 activation may lead to a decrease in IFN- $\beta$ transcription. Unfortunately, however, their experiments to assess this phenotype lacked critical controls for the efficiency of JEV infection which may itself influence IFN- $\beta$ transcription and therefore further investment of research will be required to confirm and fully explore this intriguing putative role of sfRNA.

Although the possibility that sfRNA may specifically inhibit one or more proteins involved in IFN- $\alpha / \beta$ response pathway remains open, it is probably more likely that the mechanism of inhibition of anti-viral response by sfRNA is more general and is related to the ability of sfRNA to serve as a sink for cellular RNA-binding proteins that are involved in regulation of transcription and/or translation of wide range of genes involved in various cellular response pathways, including those participating in IFN- $\alpha / \beta$ response.

\subsection{Inhibition of Host mRNA Turnover Mediated by sfRNA}

The structures that lead to sfRNA generation are highly unique as they are the first RNA elements that have been shown to consistently stall XRN1 in mammalian cells. Thus the generation of sfRNA by stalling of the XRN1 enzyme is very unusual and interestingly, has an additional perhaps highly significant impact on the cell. The generation of sfRNA results in the repression of XRN1 enzymatic activity, presumably due to the slow release of the stalled enzyme from the structures at the proximal side of the flavivirus 3'UTR [35]. The repression of XRN1 by sfRNA generation occurs with both the mammalian and mosquito enzymes, thus it is likely to impact viral infection in both the host and the vector. Furthermore, sfRNA-containing substrates directly block XRN1 enzymatic activity as repression can be observed using purified recombinant enzyme and flaviviral RNA [35]. XRN1 repression, as seen by an increase in uncapped mRNAs, occurs in infections of cells with either DENV-2 or WNV. Thus flaviviruses contain a rather novel way to shut down a host cell enzyme that is likely actively trying to degrade viral transcripts during an infection.

XRN1 repression appears to have much broader impact on the cell than simply promoting the stability of flaviviral RNAs. Approximately 400 cellular mRNAs were shown to be upregulated $3 \mathrm{X}$ or more in a WNV infection and numerous cellular mRNAs are stabilized during flavivirus infection in an sfRNA-dependent fashion due to the apparent shut down of the entire 5'-3' RNA decay pathway [35]. The feedback of the repression of XRN1 to other factors in the 5'-3' decay pathway may be due to direct protein-protein interactions between XRN1 and decapping enzymes [72] as well as through P-bodies (which, interestingly, become disrupted in flavivirus infections [46,73]). This dramatic dysregulation of cellular gene expression at the level of RNA stability by the generation of sfRNA may significantly contribute to viral pathogenesis and immune evasion.

\subsection{The RNAi Pathways and sfRNA}

RNA viruses have small genomes carrying only a minimal set of genes required for replication but also suppression of innate immune responses of their hosts. Despite their small genome size, RNA viruses have evolved unique ways to manipulate their host cell and create a specialized intracellular micro-environment to support virus replication. For RNA viruses of insects and plants, the most potent host antiviral response they counteract is RNAi [53]. The host RNAi machinery processes the viral double-stranded RNA (dsRNA) intermediates into siRNA to subsequently target and degrade the viral RNA. It is therefore no surprise that many, if not all, insect (and plant) RNA viruses encode and 
produce viral suppressors of RNAi (VSR) to inhibit antiviral RNAi. While it is clear that arboviruses suffer from RNAi [74,75] and likely have strategies to dampen the detrimental effects [76], despite efforts by different research groups arboviral VSRs have not yet conclusively been identified.

Our most recent research led to the discovery of sfRNA as a suppressor of the antiviral RNAi response in different model systems [77]. The first suggestion that WNV interfered with RNAi came from experiments that showed that induced RNAi was impaired in cells harbouring actively replicating WNV replicon RNA. Next, we initiated a screen for RNAi activity of viral products (proteins, RNA) produced during WNV replication and concluded that none of the WNV nonstructural proteins could suppress RNAi, neither in mammalian cells nor in plants. However, sfRNA was the only molecule in our screen that was capable of suppressing RNAi. Subsequent experiments showed that sfRNA displayed VSR activity in both insect as well as mammalian cells and not only affected ds/siRNA-induced RNAi, but also interfered with the miRNA pathways, again both in insect as well as mammalian cells. Interference with human Dicer processing of dsRNA in vitro suggested that sfRNA acts as a decoy molecule upstream of the RNA-induced silencing complex (RISC). As a result from this, less (antiviral) siRNA is produced when sfRNA is present, which is in line with the observation that sfRNA enhanced the replication of a heterologous arbovirus in mosquito cells [77].

The observation that sfRNA is a Dicer substrate corresponds with the production of KUN-miR-1 from the 3'UTR/sfRNA that was shown to be mediated by insect Dcr-1 [62]. The relative substrate affinity of sfRNA for insect Dcr-1 in comparison to Dcr-2, which is predominantly involved in antiviral RNAi, is currently unknown, but resolving this issue could further illuminate the precise, perhaps diverse, roles of sfRNA in insects. An attractive hypothesis to be tested is whether sfRNA production is required for efficient replication of flaviviruses in the arthropod vector, but this remains to be experimentally proven. The production of sfRNA by ISFs would certainly fit in this picture.

The exact biological activity of sfRNA as RNAi suppressor during flavivirus replication in vertebrates is still elusive, although evidence is accumulating that antiviral RNAi may exist in higher animals and humans as well [78]. In that case, sfRNA may not only feed into the endogenous miRNA pathway as we have shown [77], but could also have a profound impact on suppressing the silencing of flavivirus RNA replication in humans and other vertebrate species.

\section{Host Binding Partners of the 3'UTR and/or sfRNA}

Several candidate binding partners to the flavivirus 3'UTR have been identified via pull-down and mass-spectrometry and verified via gel shift mobility assays, immunoprecipitation, and mutational screens. Many more putative binding partners (e.g., Dicer [77]) have been inferred due to an observed functional interaction despite the lack of co-immunoprecipitation data. The binding partners variously have roles in stimulating viral gRNA replication and polyprotein translation, or function as mediators of the host anti-viral immune response. Analysis of binding partners for sfRNA specifically is distinctly lacking within the literature. Due to the conservation of sequence and structural elements between the 3'UTR and sfRNA, it is likely that many if not all of these proteins interact with this subgenomic element. Indeed due to the rapid accumulation of sfRNA it may be revealed that these proteins exert a large proportion of their functions when bound to this moiety. Table 1 summarises the identity and known functions of these 3'UTR/sfRNA binding proteins. 
Table 1. Host binding partners of the flavivirus 3'UTR and/or sfRNA.

\begin{tabular}{|c|c|c|c|c|c|c|}
\hline Protein & Origin & Function & Binds 3'UTR? & Binds sfRNA? & $\begin{array}{c}\text { Method of } \\
\text { Identification } \\
\end{array}$ & Ref. \\
\hline NS5 & Virus & Polymerase 5' RNA cap & Yes, 3'SL & Likely & $\begin{array}{l}\text { Infected cells Pull-down } \\
\text { IVT }{ }^{1} \text { RNA }\end{array}$ & {$[71,79]$} \\
\hline Capsid & Virus & Nucleocapsid & Yes & $\mathrm{NK}^{3}$, Likely & Pull-down IVT RNA & {$[80]$} \\
\hline $\mathrm{NS} 2 \mathrm{~A}$ & Virus & Viral RC Anti-IFN $^{2}$ & Yes, 3'SL & NK, Likely & IVT RNA & {$[81]$} \\
\hline NS3 & Virus & Helicase,Protease, NTPase & Yes, 3'SL & NK, Likely & IVT RNA & {$[79,82]$} \\
\hline $\mathrm{EF} 1 \alpha^{4}$ & Host & Translation elongation factor & $\begin{array}{l}\text { Yes, middle of } \\
\text { 3'SL }\end{array}$ & NK, Likely & $\begin{array}{l}\text { Infected cells, Pull- } \\
\text { down, IVT RNA }\end{array}$ & {$[24,25,83]$} \\
\hline $\mathrm{PABP}^{5}$ & Host & $\begin{array}{c}\text { Translation initiation, SG } \\
\text { component }\end{array}$ & $\begin{array}{l}\text { Yes, A-rich regions } \\
\text { flanking DBs }\end{array}$ & NK, Likely & IVT RNA & {$[23]$} \\
\hline $\begin{array}{l}\text { La } \\
\text { autoantigen }\end{array}$ & Host & $\begin{array}{l}\text { RNA chaperone, Protection } \\
\text { from RNases }\end{array}$ & Yes, 3'SL & NK, Likely & $\begin{array}{l}\text { Infected cells, Pull- } \\
\text { down, IVT RNA }\end{array}$ & {$[25,84-87]$} \\
\hline $\mathrm{PTB}^{6}$ & Host & RNA splicing & Yes & NK, Likely & Pull-down, IVT RNA & {$[25,88]$} \\
\hline DDX $6^{7}$ & Host & $\begin{array}{l}\text { PB component,Promote } \\
\text { RNA degradation }\end{array}$ & $\begin{array}{c}\text { Yes, DB1 and } \\
\text { DB2 }\end{array}$ & NK, Likely & $\begin{array}{c}\text { Infected cells, Pull- } \\
\text { down, IVT RNA, } \\
\text { Quantitative mass-spec }\end{array}$ & [89] \\
\hline Caprin $1^{8}$ & Host & $\begin{array}{l}\text { Transport and translation of } \\
\text { mRNAs, SG component }\end{array}$ & $\begin{array}{l}\text { Yes, region SL-I } \\
\text { to DB1 }\end{array}$ & $\begin{array}{l}\text { NK, Possibly } \\
\text { unless binds SL-I }\end{array}$ & $\begin{array}{l}\text { Pull-down, IVT RNA, } \\
\text { Quantitative mass-spec }\end{array}$ & [89] \\
\hline $\mathrm{G} 3 \mathrm{BP} 1 / 2^{9}$ & Host & $\begin{array}{l}\text { dsDNA or dsRNA } \\
\text { unwinding, SG components }\end{array}$ & $\begin{array}{l}\text { Yes, region SL-I } \\
\text { to DB1 }\end{array}$ & $\begin{array}{l}\text { NK, Possibly } \\
\text { unless binds SL-I }\end{array}$ & $\begin{array}{l}\text { Pull-down, IVT RNA, } \\
\text { Quantitative mass-spec }\end{array}$ & [89] \\
\hline USP10 ${ }^{10}$ & Host & $\begin{array}{c}\text { De-ubiquitination, } \mathrm{SG} \\
\text { component }\end{array}$ & $\begin{array}{l}\text { Yes, region SL-I } \\
\text { to DB1 }\end{array}$ & $\begin{array}{l}\text { NK, Possibly } \\
\text { unless binds SL-I }\end{array}$ & $\begin{array}{l}\text { Pull-down, IVT RNA, } \\
\text { Quantitative mass-spec }\end{array}$ & [89] \\
\hline FBP1 ${ }^{11}$ & Host & $\begin{array}{l}\text { ssDNA binding protein, } \\
\text { Influence mRNA stability }\end{array}$ & Yes & NK, Likely & Pull-down,IVT RNA & {$[90]$} \\
\hline p100 & Host & $\begin{array}{l}\text { Transcription and RNA } \\
\text { transport }\end{array}$ & Yes, 3'SL & NK, Likely & Pull-down,IVT RNA & {$[88]$} \\
\hline IGF2BP1 ${ }^{12}$ & Host & $\begin{array}{c}\text { Translation and mRNA } \\
\text { stability }\end{array}$ & Yes & NK, Likely & Pull-down, IVT RNA & {$[88]$} \\
\hline $\mathrm{RBMX}^{13}$ & Host & Pre-mRNA splicing & Yes & NK, Likely & Pull-down, IVT RNA & {$[88]$} \\
\hline YB-1 ${ }^{14}$ & Host & $\begin{array}{l}\text { Transcription regulation, } \\
\text { Translation regulation, } \\
\text { mRNA stability }\end{array}$ & Yes, 3'SL & NK, Likely & $\begin{array}{c}\text { Infected cells, Pull- } \\
\text { down,IVT RNA, Mass- } \\
\text { spec }\end{array}$ & [91] \\
\hline $\begin{array}{c}\operatorname{hnRNP}^{15} \\
\mathrm{Q}\end{array}$ & Host & $\begin{array}{l}\text { Splicing, Translation } \\
\text { regulation, mRNA stability }\end{array}$ & Yes & NK, Likely & $\begin{array}{c}\text { Pull-down,IVT RNA, } \\
\text { Mass-spec }\end{array}$ & [91] \\
\hline hnRNP A1 & Host & Splicing and RNA synthesis & Yes & NK, Likely & $\begin{array}{l}\text { Pull-down, IVT RNA, } \\
\text { Mass-spec }\end{array}$ & [91] \\
\hline $\begin{array}{l}\mathrm{hnRNP} \\
\mathrm{A} 2 / \mathrm{B}\end{array}$ & Host & RNA trafficking & Yes & NK, Likely & $\begin{array}{c}\text { Pull-down, IVT RNA, } \\
\text { Mass-spec }\end{array}$ & {$[91]$} \\
\hline $\operatorname{Mov} 34^{16}$ & Host & $\begin{array}{l}\text { RNA transcription and } \\
\text { translation, Proteasome }\end{array}$ & Yes, 3'SL & NK, Likely & IVT RNA & {$[92]$} \\
\hline NF90 ${ }^{17}$ & Host & $\begin{array}{l}\text { RNA export, RNA } \\
\text { stabilization, Negative } \\
\text { regulation of miRNA }\end{array}$ & Yes, 3'SL & NK, Likely & Pull-down, IVT RNA & {$[93]$} \\
\hline RHA $^{18}$ & Host & $\begin{array}{l}\text { Assist NF-kB signaling, Sense } \\
\text { dsRNA, Unwind dsRNA }\end{array}$ & $\begin{array}{l}\text { Yes, 3'SL, Maybe } \\
\text { in vitro only }\end{array}$ & NK, Possibly & Pull-down, IVT RNA & [93] \\
\hline XRN1 & Host & $\begin{array}{l}\text { PB component, } 5^{\prime}-3^{\prime} \\
\text { exoribonuclease }\end{array}$ & Yes & Yes & Infected cells,Pull-down & {$[35]$} \\
\hline
\end{tabular}

${ }^{1} \mathrm{IVT}=$ in vitro transcribed; ${ }^{2} \mathrm{IFN}=$ interferon signalling pathway; ${ }^{3} \mathrm{NK}=$ not $\mathrm{known} ;{ }^{4} \mathrm{EF} 1 \alpha=$ elongation factor $1 \alpha ;{ }^{5} \mathrm{PABP}=$ poly(A) binding protein; ${ }^{6} \mathrm{PTB}=$ polypyrimidine tract-binding protein; ${ }^{7}$ DDX6 $=$ DExD/H-box helicase $6 ;{ }^{8}$ Caprin1 = cytoplasmic activation/proliferation-associated protein 1; ${ }^{9} \mathrm{G} 3 \mathrm{BP} 1 / 2=$ GTPase-activating binding proteins 1 and $2 ;{ }^{10}$ USP10 = ubiquitin-specific peptidase 10; ${ }^{11} \mathrm{FBP} 1$ = far upstream element (FUSE)-binding protein 1; ${ }^{12}$ IGF2BP1 = Insulin-like growth factor-II mRNA-binding protein 1; ${ }^{13}$ RBMX = RNA-binding motif gene on the X chromosome; ${ }^{14} \mathrm{YB}-1=\mathrm{Y}$ box-binding protein $1 ;{ }^{15} \mathrm{hnRNP}=$ heterologous nuclear ribonucleo-protein; ${ }^{16}$ Mov34 = Moloney murine leukaemia provirus insertion-disrupted protein of $36 \mathrm{kDa} ;{ }^{17} \mathrm{NF} 90=$ nuclear factor $90 ;{ }^{18} \mathrm{RHA}=\mathrm{RNA}$ helicase A. 


\section{Conclusions and Future Directions}

Given their limited genome size, viruses naturally have to contain a high density of encoded information/activities. The sfRNA produced by flaviviruses certainly does not disappoint in this regard

as it clearly serves multiple and highly biologically-relevant roles in virus-host interactions. The sfRNA is involved in: (i) generation of miRNA, (ii) repression of a major player in cellular RNA decay, XRN1, and (iii) binding and perhaps usurping the functions of a variety of other cellular RNA binding proteins. Collectively, these functions assist the virus in evading IFN- $\alpha / \beta$ and RNAi responses and likely contribute to the replication success as well as pathogenicity of the virus in both the arthropod vector and the vertebrate host. Based on available data, the putative models of the roles of sfRNA in virus replication (Figure 4A) and in modulating the host response (Figure 4B) can thus be proposed. Three potential mechanisms of how sfRNA may modulate different processes in the flavivirus replication (Figure 4A) include:

(1) Inhibition of antigenome synthesis: sfRNA may bind to the 5'UTR of gRNA, outcompeting intermolecular interactions with the 3 'UTR and thus preventing genome cyclisation and the initiation of antigenome synthesis [20,94]. This would potentially act as a switch to increase the translation of newly synthesised positive-strand gRNA and/or packaging of gRNA into virions.

(2) Assistance in unwinding dsRNA replicative form: During the replication cycle the 5'-end of the newly completed positive gRNA must be displaced from its negative-sense partner within the double-stranded replicative form (RF). This allows the NS5 polymerase access to the 3'-end of the template antigenome $[15,95]$. sfRNA may assist in improving the efficiency of this event by exerting base-pairing interactions analogous to genome cyclisation to bind to the 5'UTR of positive-sense gRNA within the RF, thus displacing the completed strand and facilitating formation of the replicative intermediate [15]. As sfRNA is also likely to bind to elements of the viral replication complex (RC) (Table 1), sfRNA binding to $5^{\prime}$ end of $(+)$ gRNA could assist in the correct positioning of RC on the antigenome.

(3) Inhibition of translation and/or replication: sfRNA may compete with the 3'UTR of gRNA for binding to proteins of the viral RC $[20,71,79,81]$, and/or host translation machinery [23-25,96]. As sfRNA accumulates over the course of an infection it may outcompete the 3'UTR and thus act as a switch late in infection that slows replication/translation to promote gRNA packaging and viral particle assembly.

The proposed model of modulation of host response by sfRNA (Figure 4B) incorporates three so far demonstrated functions of sfRNA in (i) suppression of XRN1 activity and resultant changes in stability of cellular mRNAs which leads to disruption of cellular mRNA homeostasis, (ii) subversion of IFN- $\alpha / \beta$ signalling by an unknown mechanism leading to inhibition of anti-viral response, and (iii) suppressing the utility of cellular RNAi response likely by acting as a decoy for dicer.

The crosstalk between these three cell response pathways is of particular note (Figure 4B). XRN1 inhibition may increase the half-lives of proteins that act as negative regulators of the IFN- $\alpha / \beta$ and RNAi pathways [35]. Extensive co-regulation between the IFN- $\alpha / \beta$ and RNAi pathways has also been described, with miRNAs demonstrating control over IFN- $\beta$ expression $[92,97]$ and IFN signalling in turn modulates the profiles of cellular miRNA expression $[98,99]$. Therefore, the incomplete rescue of sfRNA-deficient mutants in a particular knock-out cell and/or mouse line may be the result of certain redundancies and/or cross-talk between various cell response pathways affected by sfRNA. Indeed it is 
likely that the observed decrease in the replication of sfRNA-deficient WNV and YFV mutants in mammalian and (especially) mosquito cell lines $[18,19,30]$ is due to an absence of the downstream effects of sfRNA antagonism of the host response (Figure 4B) leading to better cellular control of the viruses. Any intrinsic influence of sfRNA on viral replication (Figure 4A) may play a lesser role in this phenotype.

Figure 4. Proposed models of sfRNA interactions in viral replication and the host response. (A) Potential influences of sfRNA on viral gRNA replication: (i) sfRNA may prevent gRNA cyclisation, inhibiting (-) strand synthesis; (ii) sfRNA may interact via cyclisation sequences to assist in unwinding dsRNA in the replicative form at the $(+)$ strand 5 ' end, this may promote increased (+) strand synthesis; (iii) sfRNA may competitively bind and sequester translation and/or replication factors, inhibiting translation and replication of gRNA; (B) Different host response pathways inhibited by sfRNA: sfRNA interaction with XRN1 disrupts host mRNA decay; sfRNA suppresses host RNAi pathways likely through interaction with Dicer; sfRNA inhibits the IFN- $\alpha / \beta$ response via an unknown mechanism. Each of these pathways cross-communicates and may influence the activities of the others.

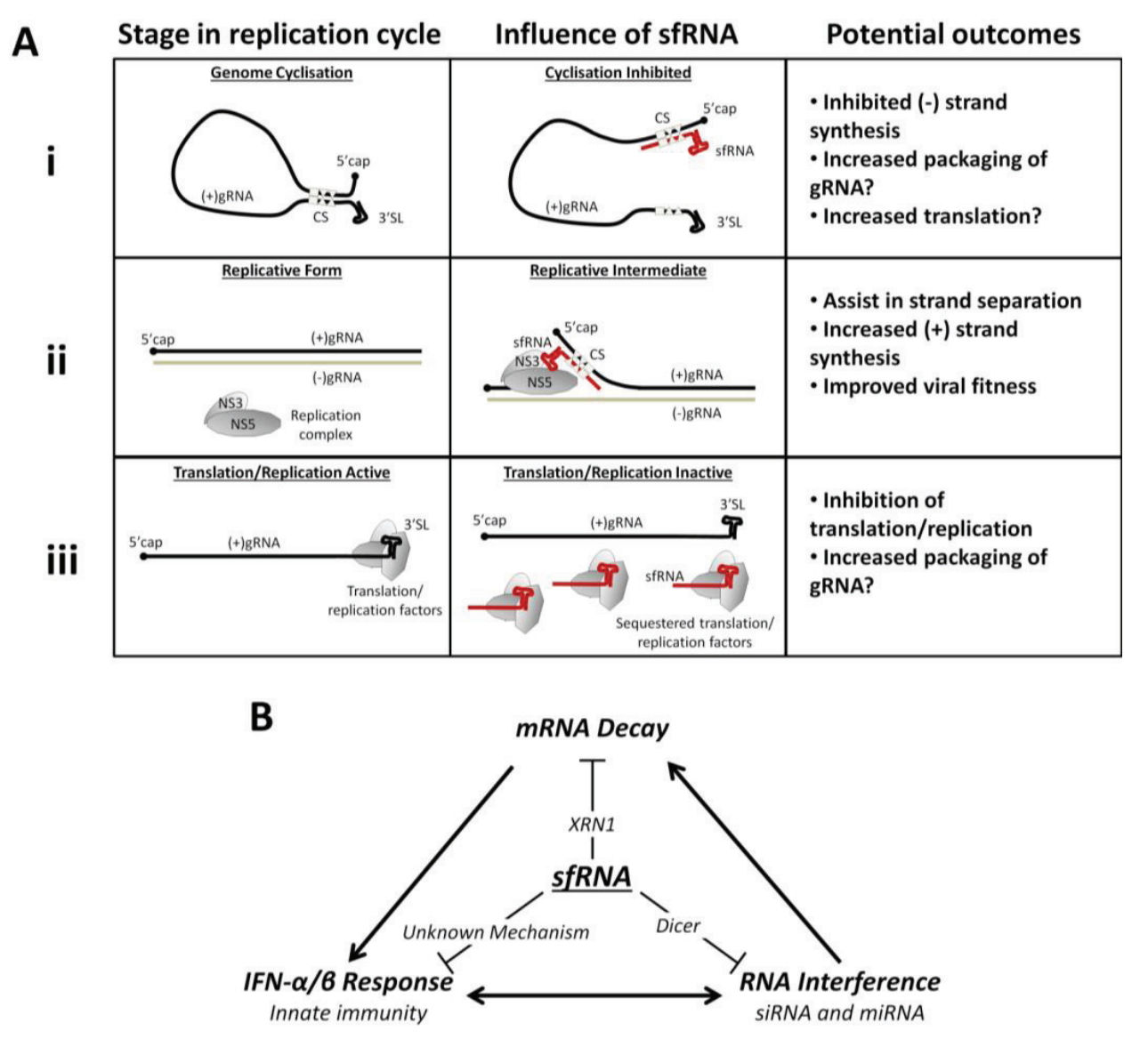

Despite rapid recent progress in identifying and characterising the mechanism of generation and functions of sfRNA, a number of interesting questions remain. The role of the sfRNA in viral interactions with the arthropod (e.g., mosquito, tick) vector needs to be investigated in more detail. Which particular nucleotide interactions in the RNA structure(s) that stalls and represses the cellular XRN1 
enzyme are essential? Is this structure unique to flaviviruses or do other viruses - or even cellular RNAs - use a similar structure to stall this exonuclease to improve mRNA stability? In addition to IFN- $\alpha / \beta$ and RNAi, can sfRNA influence other RNA-related responses in the cell? Perhaps, for example, sfRNA can serve as a sink for various cellular RNA-binding proteins during infection, dysregulating a variety of pathways in which they function. The dramatic dysregulation of cellular mRNA stability due to XRN1 repression is likely to have various pathogenic consequences that need to be elucidated. From an applied perspective, sfRNA generation may represent an interesting target for the development of broad spectrum anti-flavivirus therapeutics and attenuated phenotype of sfRNA-deficient viral mutants may prove to be very useful in developing live attenuated flavivirus vaccines. Finally, with the rising appreciation of the prominence of RNA biology and post-transcriptional gene regulation in the cell, these studies also strongly suggest that the 3'UTRs of other RNA viruses be re-examined for potentially novel functions.

\section{Acknowledgements}

We thank Peter Bredenbeek and Esther Schnettler for sharing unpublished results on sfRNA production by ISFs and TBEV, respectively. We are grateful to Mark Sterken for RNA modeling of SL-II like structures in NKVs, TBEV and TABV.

\section{Author Contributions}

J.A.R., G.P.P. and A.A.K. wrote the majority of the manuscript, J.W. wrote Section 6.3 and contributed to section 8 .

\section{Conflicts of Interest}

The authors declare no conflict of interest.

\section{References and Notes}

1. Hayes, E.B.; Gubler, D.J. West Nile virus: Epidemiology and clinical features of an emerging epidemic in the United States. In Annual Review of Medicine; Annual Reviews: Palo Alto, SA, USA, 2006; Volume 57, pp. 181-194.

2. Gyure, K.A. West Nile Virus Infections. J. Neuropathol. Exp. Neurol. 2009, 68, 1053-1060.

3. Davis, L.E.; DeBiasi, R.; Goade, D.E.; Haaland, K.Y.; Harrington, J.A.; Harnar, J.B.; Pergam, S.A.; King, M.K.; DeMasters, B.K.; Tyler, K.L. West Nile virus neuroinvasive disease. Annu. Neurol. 2006, 60, 286-300.

4. Cook, S.; Holmes, E.C. A multigene analysis of the phylogenetic relationships among the flaviviruses (Family: Flaviviridae) and the evolution of vector transmission. Arch. Virol. 2006, 151, 309-325.

5. Cook, S.; Moureau, G.; Kitchen, A.; Gould, E.A.; de Lamballerie, X.; Holmes, E.C.; Harbachl, R.E. Molecular evolution of the insect-specific flaviviruses. J. Gen. Virol. 2012, 93, 223-234.

6. Kitchen, A.; Shackelton, L.A.; Holmes, E.C. Family level phylogenies reveal modes of macroevolution in RNA viruses. Proc. Natl. Acad. Sci. USA 2011, 108, 238-243. 
7. Kuno, G.; Chang, G.J.J.; Tsuchiya, K.R.; Karabatsos, N.; Cropp, C.B. Phylogeny of the genus Flavivirus. J. Virol. 1998, 72, 73-83.

8. Gubler, D.J. Flaviviruses: Past, present and future. In Molecular Virology and Control of Flaviviruses; Shi, P.Y., Ed.; Caister Academic Press: Wymondham, UK, 2012; pp. 1-7.

9. Varelas-Wesley, I.; Calisher, C.H. Antigenic relationships of flaviviruses with undetermined arthropod-borne status. Am. J. Trop. Med. Hyg. 1982, 31, 1273-1284.

10. De Lamballerie, X.; Crochu, S.; Billoir, F.; Neyts, J.; de Micco, P.; Holmes, E.C.; Gould, E.A. Genome sequence analysis of Tamana bat virus and its relationship with the genus Flavivirus. J. Gen. Virol. 2002, 83, 2443-2454.

11. Stapleton, J.T.; Foung, S.; Muerhoff, A.S.; Bukh, J.; Simmonds, P. The GB viruses: A review and proposed classification of GBV-A, GBV-C (HGV), and GBV-D in genus Pegivirus within the family Flaviviridae. J. Gen. Virol. 2011, 92, 233-246.

12. Thurner, C.; Witwer, C.; Hofacker, I.L.; Stadler, P.F. Conserved RNA secondary structures in Flaviviridae genomes. J. Gen. Virol. 2004, 85, 1113-1124.

13. Liu, Y.; Wimmer, E.; Paul, A.V. cis-Acting RNA elements in human and animal plus-strand RNA viruses. Biochim. Biophys. Acta 2009, 1789, 495-517.

14. Lodeiro, M.F.; Filomatori, C.V.; Gamarnik, A.V. Structural and functional studies of the promoter element for Dengue virus RNA replication. J. Virol. 2009, 83, 993-1008.

15. Roby, J.A.; Funk, A.; Khromykh, A.A. Flavivirus replication and assembly. In Molecular Virology and Control of Flaviviruses; Shi, P.Y., Ed.; Caister Academic Press: Wymondham, UK, 2012.

16. Olsthoorn, R.C.; Bol, J.F. Sequence comparison and secondary structure analysis of the 3' noncoding region of flavivirus genomes reveals multiple pseudoknots. $R N A$ 2001, 7, 1370-1377.

17. Proutski, V.; Gould, E.A.; Holmes, E.C. Secondary structure of the 3' untranslated region of flaviviruses: Similarities and differences. Nucleic Acids Res. 1997, 25, 1194-1202.

18. Pijlman, G.P.; Funk, A.; Kondratieva, N.; Leung, J.; Torres, S.; van der Aa, L.; Liu, W.J.; Palmenberg, A.C.; Shi, P.Y.; Hall, R.A.; et al. A highly structured, nuclease-resistant, noncoding RNA produced by flaviviruses is required for pathogenicity. Cell Host Microbe 2008, 4, 579-591.

19. Funk, A.; Truong, K.; Nagasaki, T.; Torres, S.; Floden, N.; Melian, E.B.; Edmonds, J.; Dong, H.; Shi, P.Y.; Khromykh, A.A. RNA structures required for production of subgenomic flavivirus RNA. J. Virol. 2010, 84, 11407-11417.

20. Filomatori, C.V.; Lodeiro, M.F.; Alvarez, D.E.; Samsa, M.M.; Pietrasanta, L.; Gamarnik, A.V. A 5' RNA element promotes dengue virus RNA synthesis on a circular genome. Genes Dev. 2006, 20, 2238-2249.

21. Dong, H.P.; Zhang, B.; Shi, P.Y. Flavivirus methyltransferase: A novel antiviral target. Antivir. Res. 2008, $80,1-10$.

22. Zhang, B.; Dong, H.; Stein, D.A.; Iversen, P.L.; Shi, P.Y. West Nile virus genome cyclization and RNA replication require two pairs of long-distance RNA interactions. Virology 2008, 373, 1-13.

23. Polacek, C.; Friebe, P.; Harris, E. Poly(A)-binding protein binds to the non-polyadenylated 3' untranslated region of dengue virus and modulates translation efficiency. J. Gen. Virol. 2009, 90, 687-692.

24. Blackwell, J.L.; Brinton, M.A. Translation elongation factor-1 alpha interacts with the 3 ' stem-loop region of West Nile virus genomic RNA. J. Virol. 1997, 71, 6433-6444. 
25. De Nova-Ocampo, M.; Villegas-Sepuveda, N.; del Angel, R.M. Translation elongation factor-1 alpha, La, and PTB interact with the 3' untranslated region of dengue 4 virus RNA. Virology 2002, 295, 337-347.

26. Urosevic, N.; van Maanen, M.; Mansfield, J.P.; Mackenzie, J.S.; Shellam, G.R. Molecular characterization of virus-specific RNA produced in the brains of flavivirus-susceptible and -resistant mice after challenge with Murray Valley encephalitis virus. J. Gen. Virol. 1997, 78, 23-29.

27. Lin, K.C.; Chang, H.L.; Chang, R.Y. Accumulation of a 3'-terminal genome fragment in Japanese encephalitis virus-infected mammalian and mosquito cells. J. Virol. 2004, 78, 5133-5138.

28. Scherbik, S.V.; Paranjape, J.M.; Stockman, B.M.; Silverman, R.H.; Brinton, M.A. RNase L plays a role in the antiviral response to West Nile virus. J. Virol. 2006, 80, 2987-2999.

29. Fan, Y.H.; Nadar, M.; Chen, C.C.; Weng, C.C.; Lin, Y.T.; Chang, R.Y. Small noncoding RNA modulates Japanese encephalitis virus replication and translation in trans. Virol. J. 2011, 8, doi:10.1186/1743-422X-8-492.

30. Silva, P.A.; Pereira, C.F.; Dalebout, T.J.; Spaan, W.J.; Bredenbeek, P.J. An RNA pseudoknot is required for production of yellow fever virus subgenomic RNA by the host nuclease XRN1. J. Virol. 2010, 84, 11395-11406.

31. Liu, R.; Yue, L.; Li, X.; Yu, X.; Zhao, H.; Jiang, Z.; Qin, E.; Qin, C. Identification and characterization of small sub-genomic RNAs in dengue 1-4 virus-infected cell cultures and tissues. Biochem. Biophys. Res. Commun. 2010, 391, 1099-1103.

32. Silva, P.; Jiang, X.; Pereira, C.F.; Dalebout, T.J.; Bredenbeek, P.J. Characterization of the RNA structures required for sfRNA production in flaviviruses with no known vector. In Proceedings of the American Society of Virology 29th Annual Meeting, Montana State University, Bozeman, MT, USA, July 17-21, 2010.

33. Bredenbeek, P. Leiden University Medical Centre, Leiden, The Netherlands. Personal Communication, 2013.

34. Schnettler, E. MRC-University of Glasgow CVR, Glasgow, United Kingdom. Personal Communication, 2013.

35. Moon, S.L.; Anderson, J.R.; Kumagai, Y.; Wilusz, C.J.; Akira, S.; Khromykh, A.A.; Wilusz, J. A noncoding RNA produced by arthropod-borne flaviviruses inhibits the cellular exoribonuclease XRN1 and alters host mRNA stability. RNA 2012, 18, 2029-2040.

36. Sheth, U.; Parker, R. Decapping and decay of messenger RNA occur in cytoplasmic processing bodies. Science 2003, 300, 805-808.

37. Eulalio, A.; Behm-Ansmant, I.; Izaurralde, E. P bodies: At the crossroads of post-transcriptional pathways. Nat. Rev. Mol. Cell Biol. 2007, 8, 9-22.

38. Kedersha, N.; Stoecklin, G.; Ayodele, M.; Yacono, P.; Lykke-Andersen, J.; Fritzler, M.J.; Scheuner, D.; Kaufman, R.J.; Golan, D.E.; Anderson, P. Stress granules and processing bodies are dynamically linked sites of mRNP remodeling. J. Cell Biol. 2005, 169, 871-884.

39. Covarrubias, S.; Gaglia, M.M.; Kumar, G.R.; Wong, W.; Jackson, A.O.; Glaunsinger, B.A. Coordinated destruction of cellular messages in translation complexes by the gammaherpesvirus host shutoff factor and the mammalian exonuclease Xrn1. PLoS Pathog. 2011, 7, e1002339.

40. Markoff, L. 5'- And 3'-noncoding regions in flavivirus RNA. Adv. Virus Res. 2003, 59, 177-228. 
41. Brinton, M.A.; Fernandez, A.V.; Dispoto, J.H. The 3'-nucleotides of flavivirus genomic RNA form a conserved secondary structure. Virology 1986, 153, 113-121.

42. Shi, P.Y.; Brinton, M.A.; Veal, J.M.; Zhong, Y.Y.; Wilson, W.D. Evidence for the existence of a pseudoknot structure at the 3'-terminus of the flavivirus genomic RNA. Biochemistry 1996, 35, 4222-4430.

43. Schuessler, A.; Funk, A.; Lazear, H.M.; Cooper, D.A.; Torres, S.; Daffis, S.; Jha, B.K.; Kumagai, Y.; Takeuchi, O.; Hertzog, P.; et al. West Nile Virus noncoding subgenomic RNA contributes to Viral evasion of the type I interferon-mediated antiviral response. J. Virol. 2012, 86, 5708-5718.

44. Zhu, W.; Qin, C.; Chen, S.; Jiang, T.; Yu, M.; Yu, X.; Qin, E. Attenuated dengue 2 viruses with deletions in capsid protein derived from an infectious full-length cDNA clone. Virus Res. 2007, 126, 226-232.

45. Pahl, J.; Funk, A.; Khromykh, A. University of Queensland, Brisbane, Australia. Unpublished work, 2008.

46. Chahar, H.S.; Chen, S.; Manjunath, N. P-body components LSM1, GW182, DDX3, DDX6 and $\mathrm{XRN1}$ are recruited to WNV replication sites and positively regulate viral replication. Virology $\mathbf{2 0 1 3}, 436,1-7$.

47. Reineke, L.C.; Lloyd, R.E. Diversion of stress granules and P-bodies during viral infection. Virology 2013, 436, 255-267.

48. Fros, J.J.; Domeradzka, N.E.; Baggen, J.; Geertsema, C.; Flipse, J.; Vlak, J.M.; Pijlman, G.P. Chikungunya virus nsP3 blocks stress granule assembly by recruitment of G3BP into cytoplasmic foci. J. Virol. 2012, 86, 10873-10879.

49. Courtney, S.C.; Scherbik, S.V.; Stockman, B.M.; Brinton, M.A. West Nile virus infections suppress early viral RNA synthesis and avoid inducing the cell stress granule response. $J$. Virol. 2012, 86, 3647-3657.

50. Mackenzie, J.M.; Jones, M.K.; Young, P.R. Immunolocalization of the dengue virus nonstructural glycoprotein NS1 suggests a role in viral RNA replication. Virology 1996, 220, 232-240.

51. Welsch, S.; Miller, S.; Romero-Brey, I.; Merz, A.; Bleck, C.K.E.; Walther, P.; Fuller, S.D.; Antony, C.; Krijnse-Locker, J.; Bartenschlager, R. Composition and Three-Dimensional Architecture of the Dengue Virus Replication and Assembly Sites. Cell Host Microbe 2009, 5, 365-375.

52. Gillespie, L.K.; Hoenen, A.; Morgan, G.; Mackenzie, J.M. The Endoplasmic reticulum provides the membrane platform for biogenesis of the flavivirus replication complex. J. Virol. 2010, 84, 10438-10447.

53. Ding, S.W. RNA-based antiviral immunity. Nat. Rev. Immunol. 2010, 10, 632-644.

54. Fire, A.; Xu, S.; Montgomery, M.K.; Kostas, S.A.; Driver, S.E.; Mello, C.C. Potent and specific genetic interference by double-stranded RNA in Caenorhabditis elegans. Nature 1998, 391, 806-811.

55. Morris, K.V.; Chan, S.W.; Jacobsen, S.E.; Looney, D.J. Small interfering RNA-induced transcriptional gene silencing in human cells. Science 2004, 305, 1289-1292.

56. Brackney, D.E.; Beane, J.E.; Ebel, G.D. RNAi targeting of West Nile virus in mosquito midguts promotes virus diversification. PLoS Pathog. 2009, 5, e1000502.

57. Jinek, M.; Doudna, J.A. A three-dimensional view of the molecular machinery of RNA interference. Nature 2009, 457, 405-412.

58. Yates, L.A.; Norbury, C.J.; Gilbert, R.J. The long and short of microRNA. Cell 2013, 153, 516-519. 
59. Rouha, H.; Thurner, C.; Mandl, C.W. Functional microRNA generated from a cytoplasmic RNA virus. Nucleic Acids Res. 2010, 38, 8328-8337.

60. Varble, A.; ten Oever, B.R. Implications of RNA virus-produced miRNAs. RNA Biol. 2011, 8 , 190-194.

61. Shapiro, J.S.; Varble, A.; Pham, A.M.; Tenoever, B.R. Noncanonical cytoplasmic processing of viral microRNAs. RNA 2010, 16, 2068-2074.

62. Hussain, M.; Torres, S.; Schnettler, E.; Funk, A.; Grundhoff, A.; Pijlman, G.P.; Khromykh, A.A.; Asgari, S. West Nile virus encodes a microRNA-like small RNA in the 3' untranslated region which up-regulates GATA4 mRNA and facilitates virus replication in mosquito cells. Nucleic Acids Res. 2012, 40, 2210-2223.

63. Diamond, M.S.; Gale, M., Jr. Cell-intrinsic innate immune control of West Nile virus infection. Trends Immunol. 2012, 33, 522-530.

64. Suthar, M.S.; Diamond, M.S.; Gale, M. West Nile virus infection and immunity. Nat. Rev. Microbiol. 2013, 11, 115-128.

65. Gilfoy, F.D.; Mason, P.W. West Nile virus-induced interferon production is mediated by the double-stranded RNA-dependent protein kinase PKR. J. Virol. 2007, 81, 11148-11158.

66. Samuel, M.A.; Whitby, K.; Keller, B.C.; Marri, A.; Barchet, W.; Williams, B.R.G.; Silverman, R.H.; Gale, M.; Diamond, M.S. PKR and RNase L contribute to protection against lethal West Nile Virus infection by controlling early viral spread in the periphery and replication in neurons. J. Virol. 2006, 80, 7009-7019.

67. Elbahesh, H.; Scherbik, S.V.; Brinton, M.A. West Nile virus infection does not induce PKR activation in rodent cells. Virology 2011, 421, 51-60.

68. Tu, Y.C.; Yu, C.Y.; Liang, J.J.; Lin, E.L.; Liao, C.L.; Lin, Y.L. Blocking double-stranded RNA-activated protein kinase PKR by Japanese encephalitis virus nonstructural protein 2A. J. Virol. 2012, 86, 10347-10358.

69. Diamond, M.S.; Harris, E. Interferon inhibits dengue virus infection by preventing translation of viral RNA through a PKR-independent mechanism. Virology 2001, 289, 297-311.

70. Li, X.L.; Ezelle, H.J.; Hsi, T.Y.; Hassel, B.A. A central role for RNA in the induction and biological activities of type 1 interferons. Wiley Interdiscip Rev. RNA 2011, 2, 58-78.

71. Chang, R.Y.; Hsu, T.W.; Chen, Y.L.; Liu, S.F.; Tsai, Y.J.; Lin, Y.T.; Chen, Y.S.; Fan, Y.H. Japanese encephalitis virus non-coding RNA inhibits activation of interferon by blocking nuclear translocation of interferon regulatory factor 3. Vet. Microbiol. 2013, 166, 11-21.

72. Braun, J.E.; Truffault, V.; Boland, A.; Huntzinger, E.; Chang, C.T.; Haas, G.; Weichenrieder, O.; Coles, M.; Izaurralde, E. A direct interaction between DCP1 and XRN1 couples mRNA decapping to 5 ' exonucleolytic degradation. Nat. Struct. Mol. Biol. 2012, 19, 1324-1331.

73. Emara, M.M.; Brinton, M.A. Interaction of TIA-1/TIAR with west nile and dengue virus products in infected cells interferes with stress granule formation and processing body assembly. Proc. Natl. Acad. Sci. USA 2007, 104, 9041-9046.

74. Fragkoudis, R.; Attarzadeh-Yazdi, G.; Nash, A.A.; Fazakerley, J.K.; Kohl, A. Advances in dissecting mosquito innate immune responses to arbovirus infection. J. Gen. Virol. 2009, 90, 2061-2072.

75. Blair, C.D. Mosquito RNAi is the major innate immune pathway controlling arbovirus infection and transmission. Future Microbiol. 2011, 6, 265-277. 
76. Siu, R.W.; Fragkoudis, R.; Simmonds, P.; Donald, C.L.; Chase-Topping, M.E.; Barry, G.; Attarzadeh-Yazdi, G.; Rodriguez-Andres, J.; Nash, A.A.; Merits, A.; et al. Antiviral RNA interference responses induced by Semliki Forest virus infection of mosquito cells: Characterization, origin, and frequency-dependent functions of virus-derived small interfering RNAs. J. Virol 2011, 85, 2907-2917.

77. Schnettler, E.; Sterken, M.G.; Leung, J.Y.; Metz, S.W.; Geertsema, C.; Goldbach, R.W.; Vlak, J.M.; Kohl, A.; Khromykh, A.A.; Pijlman, G.P. Noncoding flavivirus RNA displays RNA interference suppressor activity in insect and Mammalian cells. J. Virol. 2012, 86, 13486-13500.

78. Schnettler, E.; de Vries, W.; Hemmes, H.; Haasnoot, J.; Kormelink, R.; Goldbach, R.; Berkhout, B. The NS3 protein of rice hoja blanca virus complements the RNAi suppressor function of HIV-1 Tat. EMBO Rep. 2009, 10, 258-263.

79. Chen, C.J.; Kuo, M.D.; Chien, L.J.; Hsu, S.L.; Wang, Y.M.; Lin, J.H. RNA-protein interactions: Involvement of NS3, NS5, and 3' noncoding regions of Japanese encephalitis virus genomic RNA. J. Virol. 1997, 71, 3466-3473.

80. Khromykh, A.A.; Westaway, E.G. RNA binding properties of core protein of the flavivirus Kunjin. Arch. Virol 1996, 141, 685-699.

81. Mackenzie, J.M.; Khromykh, A.A.; Jones, M.K.; Westaway, E.G. Subcellular localization and some biochemical properties of the flavivirus Kunjin nonstructural proteins NS2A and NS4A. Virology 1998, 245, 203-215.

82. Cui, T.; Sugrue, R.J.; Xu, Q.; Lee, A.K.; Chan, Y.C.; Fu, J. Recombinant dengue virus type 1 NS3 protein exhibits specific viral RNA binding and NTPase activity regulated by the NS5 protein. Virology 1998, 246, 409-417.

83. Davis, W.G.; Blackwell, J.L.; Shi, P.Y.; Brinton, M.A. Interaction between the cellular protein eEF1A and the 3'-terminal stem-loop of West Nile virus genomic RNA facilitates viral minus-strand RNA synthesis. J. Virol. 2007, 81, 10172-10187.

84. Vashist, S.; Bhullar, D.; Vrati, S. La protein can simultaneously bind to both 3'- and 5'-noncoding regions of Japanese encephalitis virus genome. DNA Cell Biol. 2011, 30, 339-346.

85. Vashist, S.; Anantpadma, M.; Sharma, H.; Vrati, S. La protein binds the predicted loop structures in the 3 ' non-coding region of Japanese encephalitis virus genome: Role in virus replication. J. Gen. Virol. 2009, 90, 1343-1352.

86. Yocupicio-Monroy, M.; Padmanabhan, R.; Medina, F.; del Angel, R.M. Mosquito La protein binds to the $3^{\prime}$ untranslated region of the positive and negative polarity dengue virus RNAs and relocates to the cytoplasm of infected cells. Virology 2007, 357, 29-40.

87. Garcia-Montalvo, B.M.; Medina, F.; del Angel, R.M. La protein binds to NS5 and NS3 and to the 5' and 3' ends of Dengue 4 virus RNA. Virus Res. 2004, 102, 141-150.

88. Lei, Y.; Huang, Y.; Zhang, H.; Yu, L.; Zhang, M.; Dayton, A. Functional interaction between cellular p100 and the dengue virus 3' UTR. J. Gen. Virol. 2011, 92, 796-806.

89. Ward, A.M.; Bidet, K.; Yinglin, A.; Ler, S.G.; Hogue, K.; Blackstock, W.; Gunaratne, J.; Garcia-Blanco, M.A. Quantitative mass spectrometry of DENV-2 RNA-interacting proteins reveals that the DEAD-box RNA helicase DDX6 binds the DB1 and DB2 3' UTR structures. RNA Biol. 2011, 8, 1173-1186. 
90. Chien, H.L.; Liao, C.L.; Lin, Y.L. FUSE binding protein 1 interacts with untranslated regions of Japanese encephalitis virus RNA and negatively regulates viral replication. J. Virol. 2011, 85, 4698-4706.

91. Paranjape, S.M.; Harris, E. Y box-binding protein-1 binds to the dengue virus 3'-untranslated region and mediates antiviral effects. J. Biol. Chem. 2007, 282, 30497-30508.

92. Ta, M.; Vrati, S. Mov34 protein from mouse brain interacts with the $3^{\prime}$ noncoding region of Japanese encephalitis virus. J. Virol. 2000, 74, 5108-5115.

93. Gomila, R.C.; Martin, G.W.; Gehrke, L. NF90 binds the dengue virus RNA 3 ' terminus and is a positive regulator of dengue virus replication. PLoS One 2011, 6, e16687.

94. Ackermann, M.; Padmanabhan, R. De novo synthesis of RNA by the dengue virus RNA-dependent RNA polymerase exhibits temperature dependence at the initiation but not elongation phase. $J$. Biol. Chem. 2001, 276, 39926-39937.

95. Chu, P.W.; Westaway, E.G. Replication strategy of Kunjin virus: Evidence for recycling role of replicative form RNA as template in semiconservative and asymmetric replication. Virology $\mathbf{1 9 8 5}$, 140, 68-79.

96. Blackwell, J.L.; Brinton, M.A. BHK cell proteins that bind to the 3' stem-loop structure of the West Nile virus genome RNA. J. Virol. 1995, 69, 5650-5658.

97. Witwer, K.W.; Sisk, J.M.; Gama, L.; Clements, J.E. MicroRNA regulation of IFN-beta protein expression: rapid and sensitive modulation of the innate immune response. J. Immunol. 2010, 184, 2369-2376.

98. Gantier, M.P.; Sadler, A.J.; Williams, B.R. Fine-tuning of the innate immune response by microRNAs. Immunol Cell Biol. 2007, 85, 458-462.

99. Pedersen, I.M.; Cheng, G.; Wieland, S.; Volinia, S.; Croce, C.M.; Chisari, F.V.; David, M. Interferon modulation of cellular microRNAs as an antiviral mechanism. Nature 2007, 449, 919-922.

(C) 2014 by the authors; licensee MDPI, Basel, Switzerland. This article is an open access article distributed under the terms and conditions of the Creative Commons Attribution license (http://creativecommons.org/licenses/by/3.0/). 